\title{
Entreprise ethnique, territorialités et filière migratoire : les migrants boliviens dans l'industrie du vêtement à São Paulo
}

\section{Patrícia Tavares de Freitas}

\section{(2) OpenEdition \\ Journals}

Édition électronique

URL : https://journals.openedition.org/e-migrinter/611

DOI : 10.4000/e-migrinter.611

ISSN : 1961-9685

Éditeur

UMR 7301 - Migrinter

Édition imprimée

Date de publication : 4 avril 2012

Pagination : 109-111

ISSN : 1961-9685

Référence électronique

Patrícia Tavares de Freitas, « Entreprise ethnique, territorialités et filière migratoire : les migrants boliviens dans l'industrie du vêtement à São Paulo », e-Migrinter [En ligne], 8 | 2012, mis en ligne le , consulté le 20 mai 2021. URL : http://journals.openedition.org/e-migrinter/611 ; DOI : https://doi.org/ 10.4000/e-migrinter.611 
 \\ Séjour de Recherche : Entreprise ethnique, territorialités et filière migratoire : les migrants boliviens dans l'industrie du vêtement à São Paulo}

\section{Patrícia Tavares de Freitas}

$\mathbf{P}$

atrícia Tavares de Freitas est une doctorante du

Département de Sociologie de 1'Université de Campinas (IFCH/Unicamp) au Brésil. Elle est impliquée dans une recherche thématique développée au Centre d'Études de Population (NEPO)

et intitulée «Observatoire des Migrations à São Paulo. Phases et aspects du phénomène migratoire ", coordonnée par la professeure Rosana Baeninger et financée par la FAPESP (Fondation pour la Recherche de 1'État de São Paulo) ainsi que par le CNPq (Conseil National du Développement Scientifique et Technologique).
Elle a passée trois mois (octobre 2011 à janvier 2012) au laboratoire MIGRINTER en tant que chercheuse invitée par Emmanuel Ma Mung dans le cadre du Programme de Financement de Court Séjour (dans les établissements de recherche à l'étranger) de la FAPESP. Ce séjour, à travers les recherches bibliographiques dans le Centre de Documentation de MIGRINTER et la participation aux activités du laboratoire, fut important et enrichissant en vue de la consolidation de l'approche théorique qu'elle développera lors de sa recherche empirique au Brésil et en Bolivie.

\section{L'immigration bolivienne et sud-coréenne liée à l'industrie du vêtement à São Paulo}

À partir de la fin des années 1980, l'environnement urbain de la ville de São Paulo a vu apparaitre la présence croissante de jeunes hommes et femmes immigrés boliviens travaillant dans des ateliers de coutures clandestins, généralement liés à la production de vêtements de commerçants coréens qui immigrèrent en Amérique Latine à partir des années 1960 .

Dans un premier temps, les sudcoréens embauchaient les Boliviens comme main-d'œuvre bon marché pour travailler au noir. Á partir des années 1990, la consolidation commerciale opérée par les Sud-coréens dans les quartiers centraux de la ville a débouché sur de nouvelles relations de travail avec les Boliviens, puisque ceux-ci accédèrent à la machinerie, au contrôle des ateliers de couture, ainsi qu'au recrutement 
de main d'œuvre depuis la Bolivie. Les sudcoréens continuèrent cependant à se charger de la conception, de la découpe des pièces du vêtement, de la fourniture de tissus et accessoires pour la production et la commercialisation.

Cette nouvelle division du travail a permis la conversion d'une partie considérable de travailleurs boliviens en petits entrepreneurs, propriétaires des ateliers de couture, participant ainsi à une hétérogénéisation de la présence bolivienne dans la ville. Pendant les années 1990, les boliviens ont commencé à gagner en visibilité dans les espaces urbains avec la formation d'associations communautaires afin d'obtenir la permission d'exercer des activités commerciales dans certains endroits spécifiques de la ville. Dès lors, des lieux tels que la Place Kantuta et la Rue Coimbra sont devenus de véritables «territoires boliviens » où i) se développent des pratiques socioculturelles qui se réfèrent à des lieux d'origine, ii) se développe une communication avec ces mêmes lieux d'origine (appels téléphoniques, envois informels de fonds, achats de billets d'avion ou d'autobus illégales pour se rendre Bolivie, etc.) iii) circulent des informations relatives au marché du travail des ateliers de la couture.

Actuellement, les petits entrepreneurs boliviens travaillent également pour les grandes multinationales telles que $C \mho A$ et Zara, mais également pour eux mêmes afin de commercialiser leur propre production dans les marchés de gros et/ou de détaillant. Bien qu'informelle, cette commercialisation participent à l'établissement d'un contrôle sur leurs propres chaines de production. Parallèlement, les boliviens rencontrent la concurrence d'autres groupes d'immigrants en provenance de pays latino-américains notamment les Paraguayens et les Péruviens -, lesquels exercent dans les mêmes activités économiques et espaces urbains.

\section{Cheminement Théorique : des « enclaves/niches ethniques " au « territoire circulatoire" et à la « médiation identitaire »}

$\mathrm{Au}$ cours de ma maîtrise, j'ai étudié l'histoire des flux migratoires boliviens et sud-coréens à destination de l'Amérique Latine depuis les années 1960. J'ai fait une recherche sur leurs contextes d'origine ainsi que sur leurs parcours migratoires. Plus précisément, dans leurs lieux de destination, j'ai pu appréhender des points de rencontre entre les Boliviens et les Sud-coréens (liées à des flux étudiés) autour des activités du secteur du vêtement à São Paulo (Brésil) et à Buenos Aires (Argentine). Dans l'objectif du doctorat, j'entreprends une recherche sur cette rencontre entre les deux groupes migratoires dans le secteur du vêtement à São Paulo en mettant l'accent sur le développement de la présence bolivienne dans ce secteur économique.

En général, les analyses dans les littératures française et anglo-saxonne - les plus connus au Brésil - sur la formation des "enclaves/niches ethniques» mettent en lumière d'un côté le rôle déterminant des nouveaux besoins de l'accumulation capitaliste dans les grandes villes conjugués au contexte de récession économique des années 1970 et 1980 ; de l'autre, l'existence des ressources ethniques spécifiques mobilisées par des immigrants entrepreneurs et leurs travailleurs.

Dans les deux cas, il s'agit donc d'examiner les déterminants économiques explicatifs de l'existence et de la permanence des «enclaves/niches ethniques», qu'ils soient externes (liés au développement socio-économique des sociétés d'accueil) ou internes (liés à des particularités des groupes ethniques étudiés). La dimension migratoire et identitaire liée aux entreprises économiques n'est généralement intégrée dans l'analyse que d'une manière secondaire. Autrement dit, tandis que d'un côté les phénomènes de mobilité ne font pas 
directement partie de l'analyse des phénomènes de sédentarité (établir une entreprise économique dans la ville ou entrer dans le marché du travail, par exemple); d'un autre côté, la dimension identitaire liée aux spécificités d'origine des immigrants est perçue d'une manière statique, comme une ressource (la ressource ethnique) à mobiliser individuellement pour leurs entreprises économiques.

Pendant mon séjour au laboratoire MIGRINTER, j'ai réalisé une recherche bibliographique dans le Centre de Documentation où j'ai pu prendre connaissance de différentes approches analytiques renouvelés mobilisant tant les faits de mobilités que les faits identitaires pour décrire les entreprises économiques des immigrés.

Dans le cadre du travail de thèse, je propose l'utilisation de deux des approches rencontrées: i) la perspective analytique développée autour du concept de territoire circulatoire proposé par Alain Tarrius ${ }^{1}$ sur les territoires établis par des groupes minoritaires (ethniques, professionnels, etc.) à partir de leurs faits de mobilité (migrations, déménagements et mobilités quotidiennes) et de sédentarité, et que traversent et subvertissent les hiérarchies locales constituées pour les projets d'aménagement et les groupes autochtones; ii) l'hypothèse de la médiation identitaire proposé pour Emmanuel $\mathrm{Ma} \mathrm{Mung}^{2}$, replaçant les entreprises économiques des immigrants dans un jeu social plus large, comme partie des stratégies de production et reproduction des individus rassemblés en tant que groupe (groupe ethnique). Ainsi, l'ensemble des entreprises économiques des groupes d'immigrants spécifiques sont perçus par Emmanuel Ma Mung comme une forme de présence collective de ces immigrants dans la

${ }^{1}$ Professeur des Universités à l'Université de Toulouse le Mirail et membre associé au laboratoire MIGRINTER et LISST-CERS.

${ }^{2}$ Directeur de recherche au CNRS et membre du laboratoire MIGRINTER. société d'accueil. Cette présence des individus en tant que groupe qui se différencie des autres déjà établis dans un même espace social suppose des processus de négociation de cette présence. Comme le propose l'auteur, cette négociation est d'abord une négociation des identités. Identités qui, dans cette approche, ne sont pas «pur héritages des identités préexistants » mais «construites à travers la négociation $»^{3}$.

De ce débat, les questions de recherche sur des entreprises économiques des Boliviens et Sud-coréens dans l'industrie du vêtement à São Paulo sont les suivantes : Quels sont les nouvelles territorialités qui émergent de leurs activités économiques, et les rapports (juxtaposition/ superposition) qui se constituent avec les territoires plus anciens développés pour l'aménagement local d'autres groupes déjà établis dans la ville? Enfin, comment se développent les processus de «négociation des identités» dans les différents moments de production et commercialisation des vêtements entre les Boliviens et les autres groupes présents dans la ville?

\author{
Patrícia Tavares de Freitas \\ Doctorante en Sociologie \\ Université de Campinas (IFCH/Unicamp - \\ Brésil) \\ tavaresdefreitas@gmail.com
}

${ }^{3}$ Cf. Ma Mung, Emmanuel. Autonomie, Migrations et Altérité. Habilitation à Diriger des Recherches (HDR de Géographie. Poitiers : Université de Poitiers, 1999, p. 196. 